\title{
The Two Reemergent Confirmed COVID-19 Cases - Manzhouli City, Inner Mongolia Autonomous Region, China, November 20, 2020
}

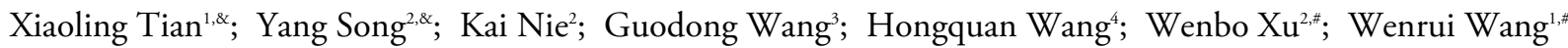

On November 20, 2020, a 55-year-old male went to the Zhongmeng Hospital of Manzhouli City, Inner Mongolia Autonomous Region for diagnosis and treatment of pain in the waist and lower extremities. His wife, a 55-year-old female, accompanied him to the hospital also feeling unwell. They were first tested for coronavirus disease 2019 (COVID-19) in Manzhouli People's Hospital based on China's policy of ensuring that "all those in need are tested, isolated, hospitalized, and treated." Later that day, Manzhouli People's Hospital reported their nucleic acid test results were positive. In the early hours of November 21 , the municipal and district-level CDCs confirmed their nucleic acid test results were positive, and after consultation with experts, comprehensive clinical examination, and inspection of imaging results, the COVID-19 diagnosis was confirmed for both patients.

On November 24, the sequences of the two Manzhouli COVID-19 patient samples were acquired using the Illumina MiSeq platform. These two Manzhouli genome sequences were identical, and when compared with the Wuhan reference sequence (EPI_ISL_ 402119) (1), 17 nucleotide variation sites were detected in the both of the Manzhouli strains, from which 8 sites (C241T, C3037T, C14408T, C18877T, A23403G, G28881A, G28882A, G28883C) were single nucleotide polymorphisms (SNPs) that belonged to the L-lineage European branch I/Pangolin lineage B.1.1 (2). Furthermore, the two Manzhouli sequences had nine unique nucleotide variation sites: C5526T, C10078T, C12952T, C14793T, C15654T, A19308G, G25650T, G29553T, and C29739T. A Russian strain was retrieved from the GISAID database (GISAID ID: EPI_ISL_5963017) that shared a total of 15 SNPs which included 7 unique SNPs with the 2 Manzhouli strains. This finding suggested it might be highly possible that the Manzhouli strains were imported from Russia. Continued monitoring of imported COVID-19 cases is vital for reducing related outbreaks (Figure 1).
The sequencing results indicated that the genomic characteristics of the Manzhouli cases were different from recent cases detected in Kashgar Prefecture, Tianjin Municipality, and Shanghai Municipality (3-5). The source of the virus was not likely a spillover event from a host or intermediate host.

Since November 21, the Manzhouli Government carried out large-scale epidemiological investigations and comprehensive nucleic acid testing. All persons that had contact with the cases or entered from the land port at Manzhouli underwent nucleic acid testing. Further investigation is still being conducted.

doi: $10.46234 / \mathrm{ccdcw} 2020.258$

\# Corresponding authors: Wenbo Xu, xuwb@ivdc.chinacdc.cn; Wenrui Wang,wr821@163.com.

\footnotetext{
${ }^{1}$ The Center for Disease Control and Prevention of Inner Mongolia, China; ${ }^{2}$ National Institute for Viral Disease Control and Prevention, China CDC, Beijing, China; ${ }^{3}$ The Center for Disease Control and Prevention of Hulunbeier City, Inner Mongolia, China; ${ }^{4}$ The Center for Disease Control and Prevention of ManZhouli County, Inner Mongolia, China.

\& Joint first authors.
}

Submitted: December 08, 2020; Accepted: December 08, 2020

\section{REFERENCES}

1. Wu F, Zhao S, Yu B, Chen YM, Wang W, Song ZG, et al. A new coronavirus associated with human respiratory disease in China. Nature 2020;579(7798):265 - 9. http://dx.doi.org/10.1038/s41586-020-2008-3.

2. Rambaut A, Holmes EC, O’Toole Á, Hill V, McCrone JT, Ruis C, et al. A dynamic nomenclature proposal for SARS-CoV-2 lineages to assistgenomic epidemiology. Nat Microbiol 2020. http://dx.doi.org/ 10.1038/s41564-020-0770-5.2.

3. Ji W, Jun Z, Xiang Z, Zhenguo G, Yang S, Xin M, et al. The first case of asymptomatic infection of COVID-19 - Kashgar Prefecture, Xinjiang Uygur Autonomous Region, China, October, 2020. China CDC Wkly 2020;2(45):882 - 883. http://dx.doi.org/10.46234/ccdcw2020.233.

4. Group C-F R, Laboratory Testing G, Yang S, Xiang Z, Xiaoyan L, Wenbo X. A case of COVID-19 - Tianjin Municipality, China, November 7, 2020. China CDC Wkly 2020;2(46):884-885. http://dx.doi.org/10.46234/ccdcw2020.241.

5. Fanghao F, Yang S, Lipeng H, Kai N, Xiaodong S. A case of COVID-19 detected in a cargo worker at Pudong Airport - Shanghai Municipality, China, November 8, 2020. China CDC Wkly 2020;2(47):910 - 911 http://dx.doi.org/10.46234/ccdcw2020.246. 


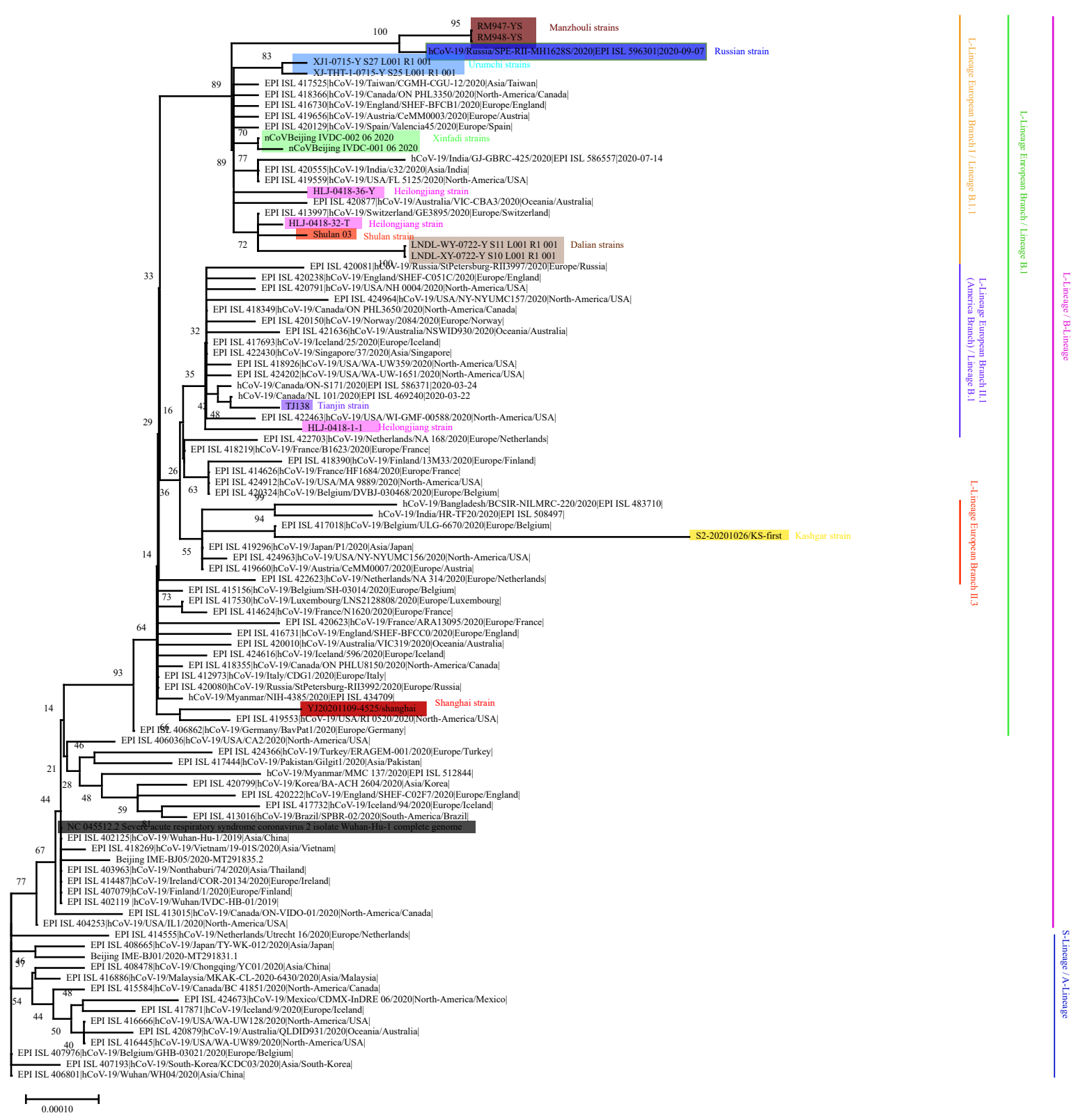

FIGURE 1. Phylogenetic tree based on the full-length genome sequences of the COVID-19 virus. The strains associated with specific outbreaks are as follows: Manzhouli City (crimson) Shanghai Municipality (red); Tianjin Municipality (purple); Kashgar Prefecture (yellow); Urumchi City (blue); Beijing Municipality Xinfadi Wholesale Market (green); northeastern China including Heilongjiang Province (pink) and Shulan (orange) related to imported cases; Dalian City (brown); Wuhan City in December 2019 (dark gray); and the Russian strain that has high nucleotide sequence homology with the Manzhouli strains (navy). The S(A)- or L(B)-lineage and sublineages of the COVID-19 virus were marked and colored on the right. 